\title{
Impulsão dinâmica da transposição da barreira. Alterações na capacidade de produção mecânica do complexo músculo- -tendinoso provocadas pela instalação da fadiga
}

\author{
Maria João Valamatos \\ Maria José Valamatos \\ Pedro Mil-Homens \\ António Veloso
}

\author{
Universidade Técnica de Lisboa \\ Faculdade de Motricidade Humana \\ Portugal
}

https://doi.org/10.5628/rpcd.05.01.15

\section{RESUMO}

O objectivo do presente estudo foi avaliar a natureza das alterações na capacidade de produção mecânica do complexo músculo-tendinoso, na fase de impulsão da transposição da barreira, induzidas pela aplicação de um protocolo de fadiga específico à prova de 400 metros barreiras. Sete barreiristas de elite nacional (idade: $24.43 \pm 5.68$ anos; altura: $1.82 \pm 0.06 \mathrm{~m}$; massa: $71.79 \pm 5.84 \mathrm{Kg}$; melhor marca $400 \mathrm{mB}: 51.55 \pm 1.72 \mathrm{~s}$ ) participaram neste estudo. A acção de transposição da barreira foi filmada com uma câmara de alta velocidade (Redlake PCI1000), que permitiu o cálculo das coordenadas bidimensionais das articulações do membro inferior. Para estimar as alterações de comprimento dos complexos músculo-tendinosos durante a fase de impulsão da transposição da barreira foram utilizadas as equações de regressão adaptadas de Jacobs et al. (14). Uma plataforma de forças tridimensional foi instalada na pista, e as forças de reacção ao apoio (Fz, Fy e Fx) foram registadas a uma frequência de $1000 \mathrm{~Hz}$ e sincronizadas com o registo de vídeo. Estes procedimentos foram repetidos antes e após a aplicação do protocolo de fadiga. As diferenças entre condições foram testadas por T-Test para amostras emparelhadas. Os principais resultados mostraram que em condições de fadiga existe um aumento significativo da perda de velocidade horizontal do Centro de Gravidade $(\mathrm{CG})(\mathrm{p}<.05)$. Este facto está associado ao aumento significativo do tempo de apoio $(\mathrm{p}<.001)$, quer do tempo da fase de amortização $(\mathrm{p}<.01)$, quer do tempo da fase de propulsão $(p<.05)$. Contudo, as forças de reacção ao apoio não registaram alterações significativas, embora tenham demonstrado uma tendência para diminuir em situação de fadiga. O aumento da duração da fase de apoio associado a alterações no comportamento angular e, consequentemente, na dinâmica muscular sugerem uma diminuição do stiffness e potência musculares em situação de fadiga.

Palavras-chave: atletismo, 400 metros barreiras, cinemática, cinética, fadiga, ciclo muscular de alongamento-encurtamento (CMAE).

\begin{abstract}
Dynamic take-off hurdles clearance. Changes on the mechanical power output variables produced by the muscle-skeletal system on the take-off phase of hurdles clearance, induced by a specially designed fatigue protocol.
\end{abstract}

The purpose of this study was to investigate changes on the mechanical power output variables produced by the muscle-skeletal system on the take-off phase of hurdles clearance, induced by a specially designed fatigue protocol, which was intended to simulate the fatigue condition of the 400 meters hurdles. Seven national elite male athletes (age: $24.43 \pm 5.68$ years old; height: $1.82 \pm 0.06 \mathrm{~m}$; body mass: $71.79 \pm$ $5.84 \mathrm{Kg} ; 400 \mathrm{mH}$ best: $51.55 \pm 1.72 \mathrm{~s}$ ) participated in this study. The performance of the clearance action was high speed video recorded at $250 \mathrm{~Hz}$ (Redlake PCI1000), and 2D coordinates of the athlete body joints were obtained. The regression equations adapted by Jacobs et al. (14) were used to estimate the length changes of the muscle tendon complex during the contact phase of the take-off of the clearance action. A three axial force platform was installed on the track, and the ground reaction forces ( $\mathrm{Fz}, \mathrm{Fy}$ and $\mathrm{Fx}$ ) were recorded synchronized with video data. These procedures were repeated before and after a fatigue protocol. The differences between both conditions were tested using TTests for paired samples. In the take-off phase of the hurdles clearance there was an increase on the loose of the horizontal velocity in the fatigue conditions $(p<.05)$. This fact was associated with the increase of the total contact time $(p<.001)$, due to a significant increase of both the breaking $(p<.001)$ and the propulsive $(p<.05)$ contact times. However, ground reactions forces did not show any sig-nificant changes. The increase of the support time and the changes in angular displacement of the joint of the trail leg, suggested a decrease in the stiffness and power output produced by the muscle-skeletal sys-tem, in the fatigue condition.

Key Words: athletics, 400 meters hurdles, kinematics, kinetics, fatigue, stretch-shortening cycle (SSC). 


\section{INTRODUÇÃO}

Os músculos ou complexos músculo-tendinosos envolvidos na mobilização dos segmentos corporais responsáveis pela locomoção humana (marcha, corrida ou saltos) estão sistematicamente sujeitos a forças de impacto com o solo, as quais condicionam um alongamento muscular. Desta forma, os músculos são inicialmente alongados por acção de uma carga externa, para depois serem activamente encurtados, produzindo movimento no sentido pretendido (18). Esta combinação constitui um tipo de comportamento muscular denominado "Ciclo Muscular Alongamento Encurtamento" (CMAE) (Stretching Shortening Cycle - SSC) $(17,18,36)$.

Estão entre este tipo de comportamento os movimentos desportivos que se caracterizam pela necessidade de realizar uma acção de impulsão, realizada através de uma extensão do membro inferior de apoio. É o caso do movimento de transposição de barreiras no atletismo, cujo apoio de impulsão se caracteriza por uma primeira fase em que o centro de gravidade do atleta está a deslocar-se no sentido descendente (fase de travagem), e à qual está associada a necessidade de alterar o sentido do deslocamento, iniciando-se, então, a segunda fase do apoio, em que o centro de gravidade do corpo do atleta é impelido no sentido pretendido (fase de propulsão). A capacidade de resistir ao alongamento muscular inicial e inverter o sentido do deslocamento de centro de gravidade resulta de aspectos mecânicos, nervosos e até metabólicos associados ao trabalho muscular e à produção de força.

Quando se fala de corrida de barreiras em atletismo, e fundamentalmente da prova de 400 metros, o rendimento está também dependente de factores associados à fadiga provocada pela acumulação de ácido láctico. Esta acumulação repercute-se numa deterioração da resposta contráctil do músculo, o que concorre para um decréscimo da intensidade de corrida e da qualidade técnica da transposição da barreira. Desta forma, a necessidade de transpor as barreiras em condições de fadiga é um aspecto determinante da prova longa de barreiras, que deve ser desenvolvido de forma a rentabilizar a performance desportiva nesta especialidade do atletismo.

Sabe-se que os movimentos que envolvem a contracção muscular em ciclo muscular de alongamento encurtamento se caracterizam por produzir, na fase de encurtamento, uma potência mecânica mais elevada do que a produzida em movimentos puramente concêntricos. Os mecanismos apontados para a explicação deste acréscimo de potência são de diverso tipo. O primeiro destes é a acumulação de energia elástica no complexo músculo-tendinoso, que acontece se a transição entre as fases excêntrica e concêntrica for realizada de forma tão breve quanto possível, e se a fase de alongamento não exceder o chamado "short range elastic stiffness" (35).

A potenciação reflexa é outro mecanismo importante que pode influenciar a produção de potência mecânica nos ciclos musculares de alongamento encurtamento. Durante a execução de movimentos que envolvam o CMAE do tipo curto, o rápido movimento excêntrico estimula o reflexo miotático ou reflexo de alongamento, desencadeado pelo fuso neuromuscular. Esta resposta aumenta a capacidade muscular de resistência ao alongamento. Ao contrário, o reflexo miotático inverso com origem nos órgãos tendinosos de Golgi, desencadeado em resposta a contracções musculares potentes, diminui esta capacidade. A relação mantida entre estes dois mecanismos define o conceito de stiffness, ou seja, a capacidade muscular de resistir a um alongamento (36).

No entanto, repetidas cargas de alongamento causam fadiga neuromuscular, que se repercute numa redução na tolerância ao alongamento, com consequente aumento do tempo de travagem e do tempo de transição entre o alongamento e o encurtamento $(10,19)$. Desta forma, os dois mecanismos referidos como responsáveis por uma potência mecânica superior vão ficando, de alguma forma, comprometidos à medida que a fadiga se instala.

Como a corrida longa de barreiras exige que as acções de impulsão sejam realizadas de igual forma, no início e no final da prova, torna-se fundamental avaliar e compreender de que forma evoluem os factores relacionados com a capacidade de produção mecânica à medida que se processa a instalação da fadiga. Assim, o propósito do presente trabalho consistiu em identificar e quantificar em que medida a necessidade de transpor barreiras em situação de fadiga neuromuscular condiciona a técnica de transposição de barreiras, no sentido de se conceberem estratégias de programação e planeamento de treino que 
visem minimizar a influência da fadiga no rendimento desportivo.

\section{MATERIAL E MÉTODOS}

A avaliação dinâmica da transposição da barreira na fase final de uma prova de $400 \mathrm{~m}$ barreiras não é exequível em situação real de competição. Por essa razão recorreu-se a uma situação experimental, que implicou a indução de fadiga e que decorreu da observação de uma competição oficial concretizada previamente. Desta forma, o presente trabalho foi desenvolvido a partir de uma concepção experimental, que importa descrever com pormenor. O quadro 1 apresenta de forma esquemática as diferentes etapas que integraram a concepção experimental.

Quadro 1: Concepção experimental.

\begin{tabular}{|c|c|c|}
\hline $1^{\circ}$ DIA & & $2^{\circ}$ DIA \\
\hline Observação de uma Competição Oficial de 400 metros Barreiras & & Situação Experimental \\
\hline $\begin{array}{l}\text { Obtenção de Parâmetros relacionados com as Intensidades de } \\
\text { Corrida }\end{array}$ & $\begin{array}{l}72 \\
\text { Horas }\end{array}$ & Avaliaçōes \\
\hline Velocidades de Corrida & & Transposiçảo da Barreira em condiçỏes de nảo Fadiga \\
\hline \multicolumn{3}{|l|}{ Ritmos de Corrida } \\
\hline Indicadores de Fadiga & & Protocolo de Fadiga \\
\hline \multirow[t]{4}{*}{ Avaliação do Lactato Sanguíneo } & & $\begin{array}{l}\text { Definição das velocidades e ritmos de corrida consoante os } \\
\text { registos de competição. }\end{array}$ \\
\hline & & Avaliação do Lactato Sanguíneo. \\
\hline & & Avaliaçōes \\
\hline & & Transposiçāo da Barreira em condiçōes de Fadiga \\
\hline
\end{tabular}

\section{Amostra}

A amostra foi composta por 7 atletas seniores masculinos (peso $71.79 \pm 5.84 \mathrm{Kg}$; altura $1.82 \pm 0.06$ m; idade $24.43 \pm 5.68$ anos; record pessoal $400 \mathrm{mB}$ $51.55 \pm 1.72 \mathrm{~s}$ ) que participaram na final da prova de 400 metros barreiras dos Campeonatos de Portugal de 2002 (6) e na final de 400 metros do mesmo Campeonato (1). Todos eles têm como especialidade principal a prova de 400 metros barreiras, tendo sido classificados entre os 10 primeiros do ranking nacional da época e entre os 25 melhores da Europa (2 deles).

\section{Acção de impulsão do movimento de transposição de barreiras}

Os indivíduos que constituíram a amostra percorreram a distância correspondente a um intervalo entre duas barreiras da corrida de 400 metros (35 metros), após uma prévia corrida de balanço (cerca de 15 metros), de forma a poder ser avaliada a acção de impulsão para a transposição da segunda barreira.
Foram gravadas imagens da acção de impulsão e registadas as forças de reacção do apoio. Estes procedimentos foram realizados antes e após a indução de fadiga, através de um protocolo específico à prova de 400 metros barreiras. De salientar que foi utilizada sempre a mesma perna de impulsão nas duas situações de avaliação.

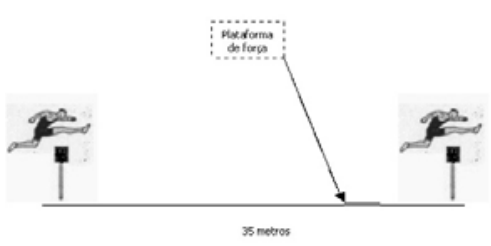

Figura 1- Figura esquemática da situação experimental utilizada no estudo. Foram recolhidas imagens e curvas de força-tempo da fase de impulsão da transposição de barreiras. Os atletas percorreram uma distância de 35 metros (intervalo entre 2 barreiras] sob condições de não fadiga e de fadiga. 


\section{Protocolo de fadiga}

Devido à impossibilidade de realizar esta avaliação em condições reais de competição, houve a necessidade de aproveitar uma unidade de treino que, pelas suas características, simula a fadiga específica da prova. Assim, o protocolo utilizado para induzir a fadiga, foi o que se descreve de seguida:

-6 séries de 2 repetições:

$1^{\text {a }}$ Repetição: 50 metros rápidos.

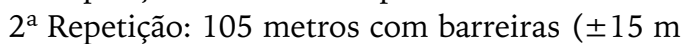
balanço; 4 barreiras com distâncias $-35 \mathrm{~m}$, e alturas $-0.91 \mathrm{~m}$, oficiais).

Estruturação:

$1^{\text {a }}$ Série - Nos $105 \mathrm{~m}$ os ritmos e velocidades aplicados foram idênticos aos realizados em situação de competição da $1^{\mathrm{a}}$ à $4^{\mathrm{a}}$ barreira;

$2^{\mathrm{a}}$ e $3^{\mathrm{a}}$ séries - ritmos e velocidades idênticos aos realizados em situação de competição da $4^{\mathrm{a}}$ à $7^{\mathrm{a}}$ barreira; $4^{\mathrm{a}}, 5^{\mathrm{a}}$ e $6^{\mathrm{a}}$ séries - ritmos e velocidades idênticos aos realizados em situação de competição da $7^{\mathrm{a}}$ à $10^{\mathrm{a}}$ barreira. - Os intervalos entre séries foram de 2 minutos e entre as repetições foi de 1 minuto.

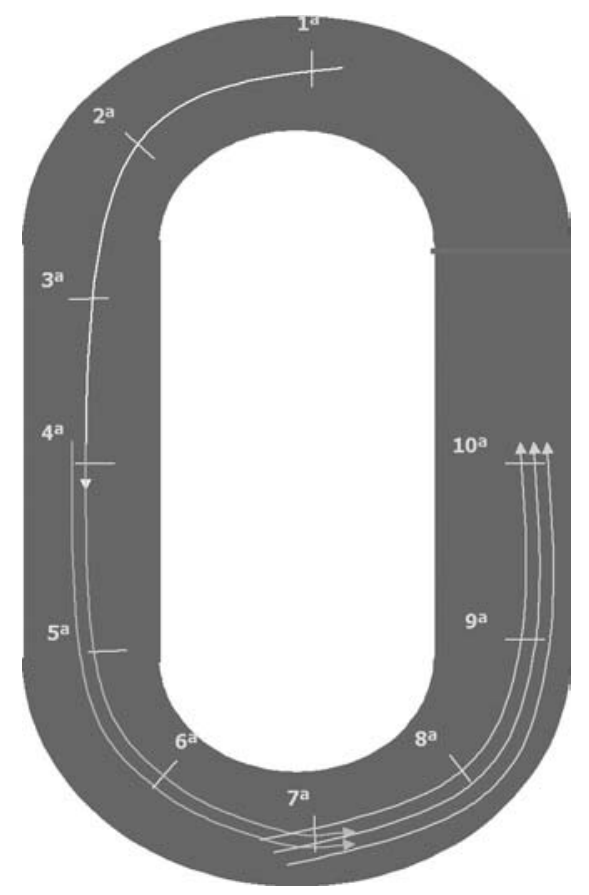

Figura 2 - Esquema da estruturação do protocolo de fadiga. Constituído por 6 séries de 4 barreiras (105m).
Para além da tentativa de garantir que velocidades e ritmos efectuados em situação experimental fossem semelhantes aos registados em situação de competição, houve também a preocupação de controlar o lactato sanguíneo em ambas as situações, de modo a garantir idêntico nível de fadiga.

As recolhas foram realizadas em intervalos de 1 minuto a partir do $4^{\circ}$ minuto após terminado o esforço. Este procedimento foi mantido até que os valores obtidos decrescessem claramente, o que nos permitiu com grande probabilidade registar o valor mais elevado de lactato sanguíneo atingido por cada um dos atletas avaliados.

\section{Instrumentos e equipamento utilizados}

$\mathrm{Na}$ zona de impulsão para a barreira foi instalada uma Plataforma de Forças (AMTI - Modelo LG 6-42000) com uma dimensão de 121,9 x $60,96 \mathrm{~cm}$, sobre a qual foi colocado um tapete de tartan. Este instrumento registou as forças de reacção do apoio a uma frequência de $1000 \mathrm{~Hz}$. As imagens foram obtidas por uma câmara de alta velocidade (MotionScope Redlake Imaging PC 1000), colocada no plano sagital da execução. A sua frequência de amostragem foi de 250 imagens por segundo. Os registos analógicos provenientes da plataforma de forças foram sincronizados com o registo de vídeo, sendo ambos os sistemas activados pelo mesmo sinal de referência.

Para controlar a velocidade das execuções foram ainda utilizados dois pares de células fotoeléctricas (Brower Timing Systems), e para controlar a velocidade do vento um anemómetro (Spring co Athletics). O controlo do lactato sanguíneo foi efectuado utilizando três "Accusport" (Boheringer Mannheim), de procedimentos analíticos simples, que permitem a obtenção fácil e rápida dos resultados a partir de uma pequena amostra sanguínea ( $\pm 15 \mathrm{ml}$ de sangue). Os registos foram armazenados num computador pessoal e os dados foram posteriormente tratados através de software específico: para a aquisição e tratamento das curvas de força foi utilizado o "Acqknowledge 3.7.2" da Biopac, e para o tratamento, digitalização e reconstrução de imagem foi utilizado o "APAS" (Ariel Performance Analysis System) da Ariel Dymanics Inc. Foram ainda utilizados os programas informáticos 
SPSS, 11.5, for Windows,, para o tratamento estatístico e o Excel para a normalização das curvas de força, cálculos relativos à dinâmica muscular e elaboração de gráficos.

\section{Procedimentos de análise e processamento de dados} Nas imagens foram recolhidas as coordenadas de 6 pontos anatómicos que formam um modelo composto por quatro corpos rígidos: pé, perna, coxa e o conjunto tronco, cabeça e membros superiores. No âmbito deste trabalho, este conjunto tomou a designação de tronco. Os pontos anatómicos considerados foram os seguintes:

ponto 1: centro da articulação do metatarso com a $1^{\mathrm{a}}$ falange;

ponto 2: zona posterior inferior do calcâneo; ponto 3: articulação tibio-társica ao nível da linha inter maleolar; ponto 4: articulação do joelho ao nível da fenda arti- cular tíbio-femoral;

ponto 5: articulação coxo-femoral ao nível do centro do acetábulo;

ponto 6: articulação escapulo-umeral ao nível do centro da cavidade glenóide.

Como factor de conversão do valor das coordenadas em valores reais, foi utilizado um referencial de calibração com 8 pontos ocupando uma área de $1.16 \mathrm{x}$ $1.69 \mathrm{~m}$. As reconstruções de imagem foram realizadas no sistema de tratamento de imagem através do método DLT (Direct Linear Transformation) para duas dimensões. A digitalização foi realizada manualmente, iniciando-se este processo 10 imagens antes do início do apoio e terminando 10 imagens após o final do contacto. A suavização dos dados cinemáticos foi realizada por filtragem digital, tendo sido utilizado um filtro digital a uma frequência de corte de $18 \mathrm{~Hz}$.

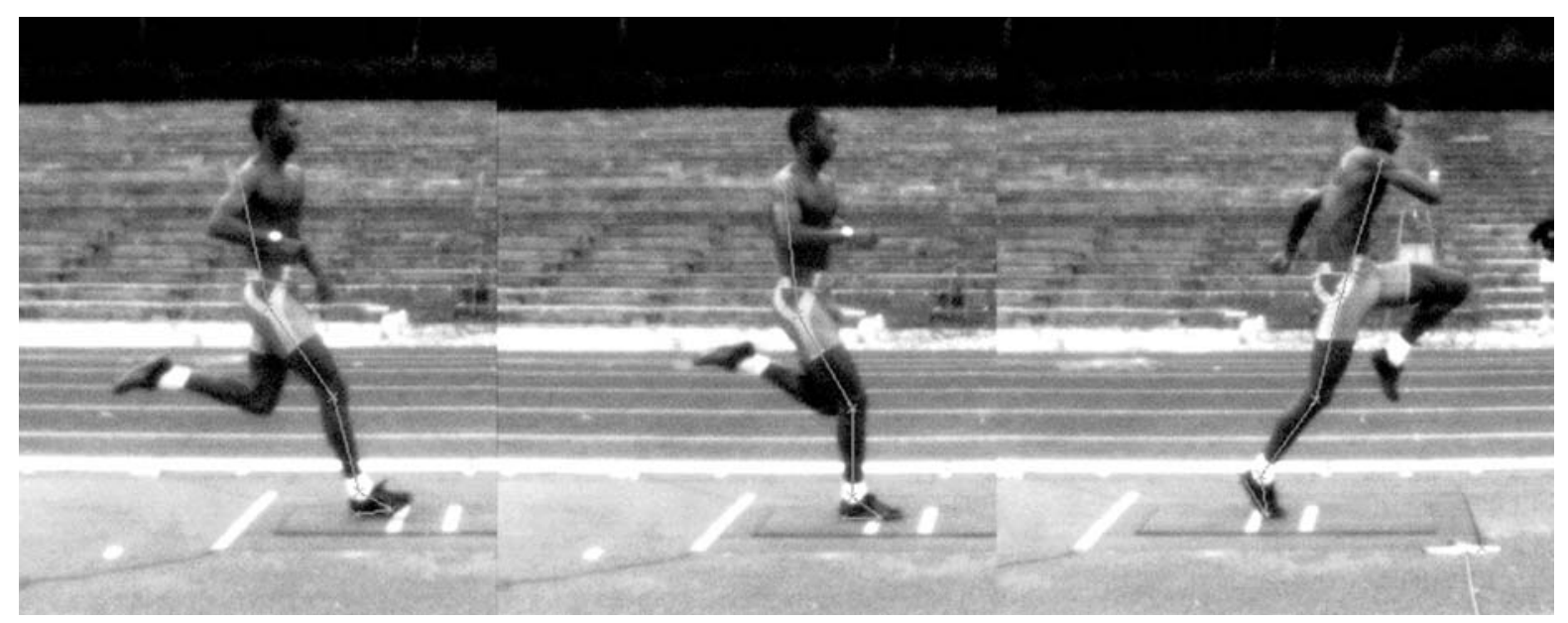

Figura 3 - Imagens sobre as quais estão projectados os segmentos de recta que representam os corpos rígidos definidos para o modelo. As imagens apresentadas correspondem à primeira imagem em apoio (imagem da esquerda), imagem em que se regista a maior flexão do joelho (imagem do meio] e a última imagem em apoio (imagem da direita), da acção de impulsão da transposição de barreiras.

O conhecimento das posições articulares instantâneas permitiu determinar as variações de comprimento dos músculos que cruzam essas articulações. As características do comportamento muscular têm sido estudadas em condições in vitro a partir de trabalhos realizados em cadáveres humanos, permitindo que as variações registadas no comprimento do músculo sejam estima- das a partir do conhecimento das variações angulares das articulações $(11,15,41)$. As equações de predição encontradas nestes estudos são normalizadas em função do comprimento dos segmentos corporais dos cadáveres, permitindo, desta forma, generalizá-las e aplicá-las a outros sujeitos, desde que o comprimento dos segmentos seja considerado nos cálculos (40). 
Para estimar a variação de comprimento instantâneo dos complexos músculo-tendinosos do membro inferior analisados neste trabalho foram utilizadas as equações polinomiais preditivas determinadas por Visser e col. (41) e as adaptações e compilações realizadas por Jacobs e Schenau (15).

O conhecimento da variação de comprimento muscular instantâneo permitiu distinguir as fases de acção excêntrica e concêntrica dos complexos músculo-tendinosos estudados e analisar a duração e o comportamento muscular em cada uma delas. Esta separação de fases de acção muscular permitiu também o cálculo das velocidades de alongamento e encurtamento registadas em cada execução e para cada um dos músculos avaliados.

Para a comparação intra e inter individual os ficheiros representativos do comportamento muscular em cada execução foram normalizadas em tempo, para $100 \%$ da fase de apoio, tomando como referência o ficheiro com menor número de amostras. Os procedimentos utilizados para esta normalização foram os recomendados por Cabri (4), que permitem retirar amostras de forma intervalada, sem alterar a sua forma. Para além disso, permitem realizar a comparação entre as várias execuções e ter uma apreciação do comportamento global do sistema músculoesquelético estudado (40).

Os dados obtidos das forças de reacção do apoio foram também analisados, tendo sido considerados para estudo os valores individuais obtidos nas variáveis estudadas. Posteriormente, para determinação das curvas médias, os ficheiros representativos de cada acção foram normalizados em tempo para $100 \%$ da fase de apoio, respeitando os procedimentos anteriormente referidos.

\section{Análise estatística}

Procedimentos estatísticos descritivos foram utilizados para caracterizar a amostra e os valores das diferentes variáveis em termos de tendência central (média aritmética) e de dispersão absoluta (desvio-padrão). O tratamento estatístico de comparação de valores médios relativos às diferentes variáveis analisadas foi realizado através de t-test's. Quando verificada a distribuição normal para as diferenças entre condições ( $\mathrm{p}>0.05)$, recorreu-se à aplicação do teste paramétrico Paired-Sample Test e quando verificada a ausência de distribuição normal $(\mathrm{p}<0.05)$, foi realizado o teste não paramétrico de Wilcoxon. O grau de confiança escolhido para os valores estatisticamente significativos foi de $95 \%$.

No sentido de avaliar o grau de associação das diferentes variáveis foi utilizado o coeficiente de correlação (Pearson correlation coefficients).

\section{RESULTADOS}

A efectividade do protocolo de fadiga (PF) foi verificada através da comparação da concentração de lactato sanguíneo (CLS) obtida no final deste protocolo com a concentração de lactato sanguíneo na prova dos Campeonatos Nacionais (CN), realizada três dias antes. Foram comparados os valores de quatro dos sete atletas testados, tendo o teste estatístico de comparação de médias revelado concentrações idênticas (CLS: CN 17.83 \pm 3.12 ; PF $17.25 \pm 3.17$, p-value $0.15)$. Deste modo, podemos afirmar que o protocolo de fadiga seleccionado permite recriar, em termos de concentração de lactato sanguíneo, a fadiga inerente à prova de 400 metros barreiras.

Dos factores que caracterizam uma prova de velocidade, a capacidade de aplicar níveis de força elevados em curtos períodos de tempo apresenta-se como uma das principais condições de rendimento. No presente estudo, o tempo necessário para aplicar os níveis de força necessários à transposição foi inferior a 150 milésimos de segundo. A figura 4 apresenta a duração da fase de impulsão da transposição da barreira nas duas condições estudadas e permite verificar que em condições de fadiga existiu um aumento significativo do tempo de impulsão.

\section{Tempo de Apoio \\ (ms)}

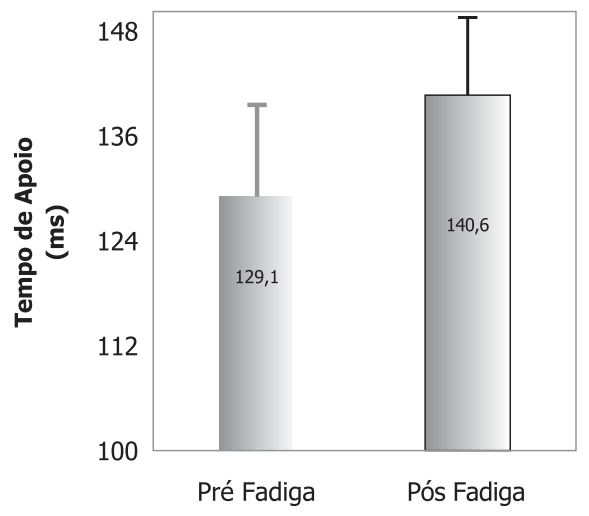

Figura 4-Duração do apoio de impulsão nas duas condições estudadas. ${ }^{* *} p<0.001$. 


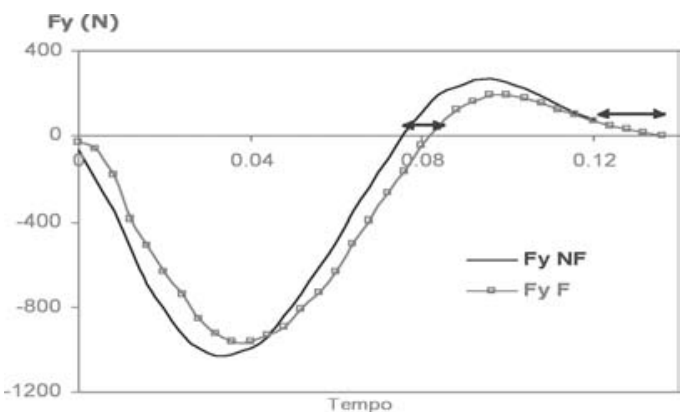

Figura 6 - Curvas de força-tempo da componente antero-posterior (Fy) produzidas por um dos atletas que integraram a amostra, obtidas em condições de não fadiga (NF) e de fadiga $(F)$.

O facto do impulso horizontal total ser negativo induz, logo à partida, uma diminuição significativa da velocidade horizontal do CG, desde o início do apoio, até que o atleta abandona o contacto com solo. Este facto não é exclusivo do apoio realizado em condições de fadiga, registando-se também uma perda de velocidade durante a execução sem fadiga. Contudo, e como se pode observar no quadro 4 , a perda de velocidade durante o apoio é significativamente superior em condições de fadiga. Esta diminuição da velocidade horizontal condiciona de forma significativa a distância à barreira a que é realizado o apoio de impulsão (distância de take-off). Esta apresenta uma diminuição da ordem dos $4 \%$.

Quadro 4 - Velocidade Horizontal do CG à entrada e à saída do apoio de impulsão para a transposição, variação da velocidade horizontal ( $\Delta V$ CG) durante $o$ apoio e perda relativa da velocidade horizontal nas duas condições estudadas: não fadiga (NF) e fadiga (F). São apresentados os valores de média, desvio-padrão (DP] e nível de significância estatística (NSE].

\begin{tabular}{|c|c|c|c|c|c|}
\hline \multirow[b]{2}{*}{ Parâmetro } & \multicolumn{2}{|c|}{ Não Fadiga (NF) } & \multicolumn{2}{|c|}{ Fadiga (F) } & \multirow[b]{2}{*}{ NSE } \\
\hline & Média & DP & Média & DP & \\
\hline \multicolumn{6}{|l|}{ Velocidade } \\
\hline Horizontal do & 8.96 & 0.65 & 8.35 & 0.54 & $p<$ \\
\hline CG à entrada & & & & & 0.005 \\
\hline \multicolumn{6}{|l|}{ do apoio } \\
\hline \multicolumn{6}{|l|}{$\left(\mathrm{ms}^{-1}\right)$} \\
\hline \multicolumn{6}{|l|}{ Velocidade } \\
\hline Horizontal do & 8.71 & 0.50 & 7.78 & 0.33 & $p<$ \\
\hline CG à saída do & & & & & 0.001 \\
\hline \multicolumn{6}{|l|}{ apoio } \\
\hline \multicolumn{6}{|l|}{$\left[\mathrm{ms}^{-1}\right]$} \\
\hline \multicolumn{6}{|l|}{ Perda de } \\
\hline Velocidade & 2.64 & 4.40 & 6.64 & 3.98 & $p<$ \\
\hline Horizontal & & & & & 0.05 \\
\hline \multicolumn{6}{|l|}{$(\%)$} \\
\hline Distância de & 2.31 & 0.24 & 2.22 & 0.21 & $\mathrm{p}<$ \\
\hline Take-off $(\mathrm{m})$ & & & & & 0.05 \\
\hline
\end{tabular}

As condições de take-off resultam do comportamento articular durante o apoio que permite travar o movimento descendente do CG e promover o seu reenvio posterior no sentido ascendente, utilizando a energia acumulada nas estruturas muscular e tendinosa.

O quadro 5 apresenta os resultados cinemáticos angulares médios obtidos pelos 7 atletas. São indicados os valores médios e os desvios-padrão para cada parâmetro. É também indicada a percentagem do tempo de apoio em que ocorrem a máxima dorsiflexão, a velocidade máxima de dorsiflexão, a flexão máxima do joelho e as velocidades máximas de extensão da tíbiotársica, do joelho e da coxo-femoral.

É ainda possível observar, a partir do quadro 5, uma sequência próximo-distal de ocorrência das velocidades máximas de extensão das três articulações. Assim, o valor máximo de extensão da coxo-femoral ocorre cerca dos $71 \%$ do tempo de apoio, seguido do pico máximo de velocidade de extensão do joelho, que ocorre aos $76 \%$ da fase de apoio e, finalmente, a articulação tíbio-társica atinge a sua velocidade máxima de plantarflexão próximo dos $81 \%$ do tempo de contacto. Este comportamento mantém-se em condições de fadiga, embora os picos de velocidade de extensão sejam ligeiramente inferiores e atingidos mais tarde, reflectindo uma diminuída potência dos músculos envolvidos na acção de transposição da barreira. 
Quadro 5 - Valores médios e desvios-padrão das posições angulares de contacto e de saída do apoio para as articulações da tíbio-társica, joelho e coxo-femoral, nas duas condições estudadas: Não Fadiga (NF) e Fadiga (F). São também apresentados os valores de velocidade máxima de extensão das três articulações e de dorsiflexão da tíbio-társica e a percentagem do tempo de apoio em que esse valor ocorre.

\begin{tabular}{|c|c|c|c|c|c|c|}
\hline \multirow[b]{2}{*}{ Tíbio-Társica } & \multicolumn{3}{|c|}{ Não Fadiga (NF) } & \multicolumn{3}{|c|}{ Fadiga $[F]$} \\
\hline & Média & DP & $\%$ T.A. & Média & DP & \% T.A. \\
\hline$\theta$ de contacto (rad) & 1.74 & 0.13 & & 1.77 & 0.17 & \\
\hline$\theta$ mínimo (rad) & 1.34 & 0.07 & 48.3 & 1.38 & 0.06 & 47.9 \\
\hline$\theta$ de saída (rad] & 2.19 & 0.11 & & 2.26 & 0.11 & \\
\hline$\varpi_{\max }$ dorsiflexão $\left(\mathrm{rad} \mathrm{s}^{-1}\right)$ & -9.65 & 2.31 & 24.6 & -9.34 & 1.85 & 24.6 \\
\hline$\Phi_{\max }$ plantarflexão $\left(\mathrm{rad} \mathrm{s}^{-1}\right)$ & 18.36 & 3.06 & 81.4 & 17.38 & 2.30 & 80.9 \\
\hline \multicolumn{7}{|l|}{ Joelho } \\
\hline$\theta$ de contacto (rad] & 2.78 & 0.05 & & 2.80 & 0.08 & \\
\hline$\theta$ mínimo (rad) & 2.44 & 0.09 & 48.3 & 2.41 & 0.12 & 51.4 \\
\hline$\theta$ de saida (rad) & 2.81 & 0.06 & & 2.78 & 0.16 & \\
\hline $\bar{\varpi}_{\text {max }}$ extensão $\left[\mathrm{rad} \mathrm{s}^{-1}\right]$ & 8.43 & 2.14 & 76.4 & 8.42 & 1.81 & 78.6 \\
\hline \multicolumn{7}{|l|}{ Coxo-femoral } \\
\hline$\theta$ de contacto (rad) & 2.65 & 0.14 & & 2.67 & 0.15 & \\
\hline$\theta$ de saida (rad) & 3.21 & 0.16 & & 3.13 & 0.05 & \\
\hline $\bar{\varpi}_{m j x}$ extensão $\left[\mathrm{rad} \mathrm{s}^{-1}\right]$ & 9.19 & 3.54 & 71.1 & 8.27 & 1.63 & 75.4 \\
\hline
\end{tabular}

Como anteriormente referido, a variação das trajectórias e posições angulares das articulações do membro inferior de apoio provoca alterações no comportamento muscular.

É importante referir que, no movimento de impulsão para a transposição da barreira, as acções dos músculos extensores do joelho e plantarflexores apresentam claros ciclos musculares de alongamento encurtamento. Por sua vez, os músculos extensores da coxo-femoral (isquiotibiais) não apresentam fase de alongamento, mas apenas uma contracção concêntrica. Este comportamento resulta da influência das duas articulações por eles cruzadas, a coxo-femoral e o joelho. No início do contacto, a coxo-femoral permanece quase inalterada, sendo o comportamento dos músculos Isquiotibiais (ISQ) influenciado essencialmente pela flexão do joelho, que provoca o seu encurtamento. Quando se inicia a extensão do joelho, verifica-se também uma extensão da coxo-femoral. A primeira provoca um alongamento dos ISQ e a segunda o seu encurtamento. Ainda assim, e pelo facto de ser essencialmente um extensor da coxofemoral, existe uma contraç̧ão concêntrica dos ISQ.
Como se pode observar nas figuras 7 e 8 , os músculos extensores do joelho (o Vastos - VL e o Recto Femoral - RF) e os músculos plantarflexores (Gémeos - GM e Solear - SOL) apresentam um claro comportamento em ciclo muscular de alongamento encurtamento do tipo curto, com velocidades de alongamento elevadas e uma amplitude de alongamento mínima.

De notar que os GM nunca atingem valores positivos, o que quer dizer que durante a fase de apoio da transposição da barreira os GM estão sempre mais encurtados relativamente à "posição de alinhamento vertical" (40), que corresponde a um ângulo da tíbio-társica de $90^{\circ}$ e do joelho e coxo-femoral de $180^{\circ}$. Assim, a conjugação dos comportamentos do joelho e da tíbio-társica durante o movimento de impulsão na transposição da barreira diminui a amplitude de movimento dos gémeos, o que explica também os valores inferiores de velocidade de contracção dos GM. 


\section{$\Delta$ C. RF (m)}

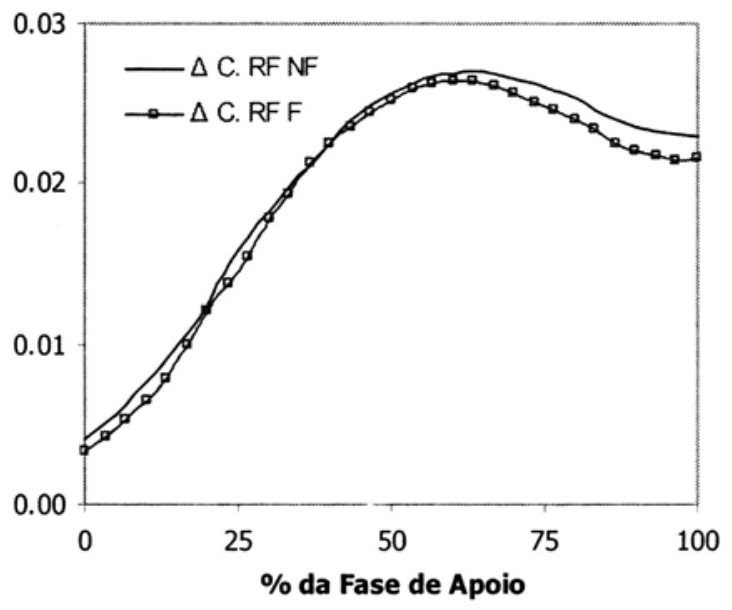

$\Delta$ C. VL (m)

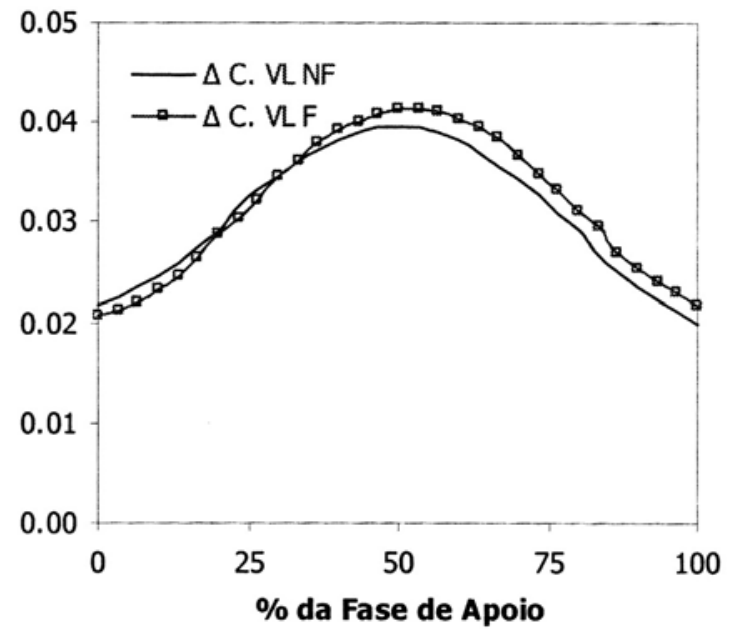

Figura 7 - Curvas médias de variação do comprimento ( $\Delta C$.) do recto femoral (RF] e do vastos. 0 tempo é expresso em percentagem da fase de apoio.

\section{$\Delta$ C. GM (m)}

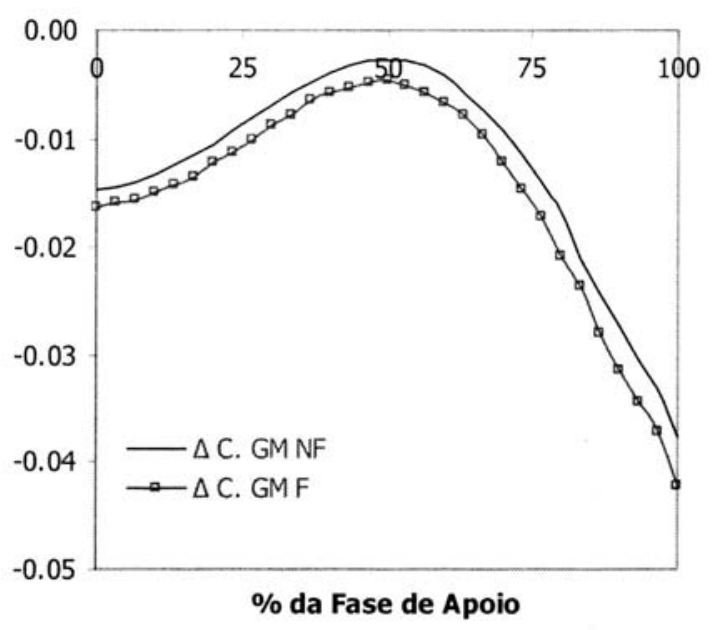

$\triangle$ C. SOL (m)

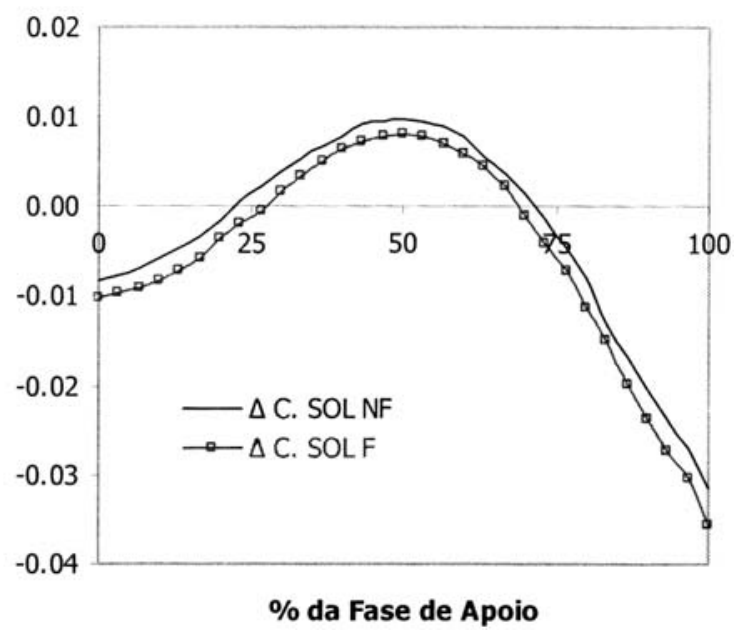

Figura 8 - Curvas médias de variação do comprimento ( $\Delta C$.] dos Gémeos (GM] e do Solear [SOL]. 0 tempo é expresso em percentagem da fase de apoio.

A influência da fadiga nestes comportamentos faz-se sentir essencialmente na maior duração e variação excêntrica do VL e na maior duração e variação concêntrica do RF. Da observação do quadro 6 é possível verificar que a amplitude do alongamento do tricípite sural não registou alterações significativas. No entanto, o superior tempo de apoio conduziu a um aumento da duração das fases de contracção excêntrica e concêntrica dos complexos músculo-tendinosos. Deste modo, o facto do aumento da duração da fase excêntrica não ter sido acompanhada pelo aumento da amplitude do alongamento implicou uma velocidade de alongamento inferior, o que sugere uma diminuída potenciação reflexa. 
Quadro 6 - Variação de comprimento muscular e duração das fases de alongamento e encurtamento dos músculos Gémeos [GM] e Solear [SOL] nas duas condições estudadas: não fadiga (NF) e fadiga (F). São apresentados os valores de média, desvio-padrão (DP) e nível de significância estatística (NSE).

\begin{tabular}{|c|c|c|c|c|c|}
\hline \multirow[b]{2}{*}{ Parâmetro } & \multicolumn{2}{|c|}{ Não Fadiga (NF) } & \multicolumn{2}{|c|}{ Fadiga (F) } & \multirow[b]{2}{*}{ NSE } \\
\hline & Média & DP & Média & DP & \\
\hline \multicolumn{6}{|l|}{ GM } \\
\hline Tempo Excêntrico (ms) & 62.8 & 6.0 & 65.1 & 7.6 & NS \\
\hline Variação Excêntrica (mm) & 12.1 & 4.3 & 12.4 & 4.9 & NS \\
\hline Tempo Concêntrico (ms] & 66.3 & 6.9 & 74.3 & 7.6 & $p<0.05$ \\
\hline Variação Concêntrica (mm) & 35.0 & 5.2 & 37.7 & 4.9 & $p<0.05$ \\
\hline \multicolumn{6}{|l|}{ SOL } \\
\hline Tempo Excêntrico (ms) & 62.9 & 5.5 & 67.4 & 6.7 & $p<0.05$ \\
\hline Variação Excêntrica (mm) & 18.3 & 5.1 & 18.9 & 5.7 & NS \\
\hline Tempo Concêntrico (ms) & 66.3 & 6.5 & 73.1 & 6.4 & $p<0.01$ \\
\hline Variação Concêntrica (mm) & 41.4 & 6.1 & 44.0 & 5.0 & $p<0.01$ \\
\hline
\end{tabular}

Por outro lado, a superior extensão da articulação tíbio-társica em fadiga provoca um aumento da amplitude de encurtamento do GM e do SOL. Como a duração da fase de encurtamento também aumentou, a velocidade de contracção não registou alterações significativas em situação de fadiga, embora apresente uma ligeira tendência de diminuição, em resultado do aumento mais significativo da duração da fase de encurtamento.

\section{DISCUSSÃO}

Cinética do apoio de impulsão da transposição da barreira São vários os autores que sugerem que a fase de impulsão da transposição de barreiras, no que diz respeito às forças de reacção do apoio, se assemelha em grande escala à fase de impulsão do salto em comprimento $(24,28)$, apresentando, no entanto, magnitudes menores. Segundo Molina e Oscariz (28), a força produzida no apoio de impulsão para o salto em comprimento alcança os 7BW, enquanto que no apoio de impulsão para a transposição da barreira atinge os 5BW. No entanto, quando referem este valor, os autores não mencionam a altura da barreira, podendo referir-se à altura de $1.06 \mathrm{~m}$ (110mb) ou à de $91.4 \mathrm{~cm}$ (400mb). Já McLean (26) avalia a transposição da barreira com uma altura de $1.06 \mathrm{~m}(110 \mathrm{~m})$ registando valores máximos de força vertical na ordem dos 5.72BW.
A transposição de uma barreira com uma altura de $91.4 \mathrm{~cm}$ não exige, provavelmente, a mesma magnitude de força que outra com $1.06 \mathrm{~m}$, pelo que os valores obtidos na presente avaliação se enquadram nos valores de referências existentes na literatura. Os valores encontrados no presente estudo são idênticos aos registados em apoios de corridas de velocidade, que atingem os 4.3BW a velocidades máximas de $9.23 \mathrm{~ms}^{-1}$ (33), ou os $4.6 \mathrm{BW}$ a velocidades supramáximas, induzidas através de um sistema de "corrida assistida" (27).

Aliás, os valores máximos da componente vertical das forças de reacção do apoio correlacionam-se directamente com a velocidade de corrida $(16,32)$, podendo alcançar entre os $2.5 \mathrm{BW}$ (16) e os $2.9 \mathrm{BW}$ (32) a velocidades de $6.0 \mathrm{~ms}^{-1}$, e os $4.6 \mathrm{BW}$ a velocidades supramáximas (27).

Sendo os 400 metros barreiras uma prova máxima, realizada a velocidades elevadas, os valores encontrados no nosso estudo pressupõem diferenças mínimas relativamente a um apoio normal da corrida entre as barreiras.

Contrariamente ao que acontece num apoio de corrida, as forças de reacção do apoio na impulsão para a barreira não diminuem significativamente em condições de fadiga. Efectivamente, em estudos que analisam os efeitos da fadiga na corrida de 400 metros são registados valores significativamente 
menores da componente vertical das FRA na parte final da corrida (33).

O apoio de impulsão, assim como qualquer apoio de corrida, caracteriza-se por integrar uma primeira fase de travagem e uma segunda fase de impulsão ou propulsão. A primeira acontece porque o pé está a mover-se para a frente (horizontalmente) em relação ao solo no momento que precede o apoio.

Efectivamente, no momento de contacto do pé com o solo, o CG está a deslocar-se no sentido descendente, sendo necessário alterar esse sentido durante o apoio. A orientação das forças de reacção do apoio é, então, consequência das posições angulares das diferentes articulações que permitem travar o movimento descendente do CG e promover o reenvio posterior no sentido ascendente, utilizando a energia acumulada nas estruturas muscular e tendinosa. O sucesso e eficiência do apoio de corrida ou de impulsão relaciona-se com a minimização da duração e dimensão da fase de travagem e a optimização da duração e dimensão da fase de propulsão. No entanto, a curva da componente antero-posterior das forças de reacção do apoio registadas na impulsão para a transposição da barreira caracteriza-se por apresentar um grande impulso de travagem, seguido de um pequeno impulso de propulsão (24).

Os resultados obtidos no presente estudo estão de acordo com McDonald e Dapena (24). Contudo, e à semelhança do que ocorre na componente vertical das forças de reacção do apoio, não se registaram alterações significativas nestes parâmetros, apesar da literatura referir que as forças de reacção do apoio diminuem significativamente com a instalação da fadiga (33). Estes resultados permitem concluir que a transposição da barreira requer, provavelmente, um determinado nível de força, sem o qual estaria comprometida. Esta hipótese é suportada pelos resultados obtidos nos parâmetros verticais e horizontais das FRA, que, ao permanecerem inalterados com a instalação da fadiga, permitem presumir que a grande diferença entre as duas condições não está nos níveis de força aplicados, mas sim no tempo necessário para atingi-los. Deste modo, o aumento significativo do tempo de impulsão poderá ser interpretado como uma tentativa de compensar uma diminuída capacidade muscular, evitando assim diminuições significativas da força.

No entanto, e ainda que não tenham sido registadas diminuições nos valores da força aplicada no solo, a verdade é que a distância de impulsão diminuiu significativamente em condições de fadiga, sugerindo que, embora os atletas apliquem idênticos níveis de força, não conseguem uma performance idêntica. Por outro lado, a fadiga está também relacionada com as perdas de velocidade horizontal durante o apoio. Os resultados obtidos no presente estudo estão de acordo com os registados no estudo de Sprague e Mann (38) que registaram aumentos significativos da perda de velocidade durante o apoio na parte final de uma competição de 400 metros. Estas perdas de velocidade horizontal estão relacionadas com o aumento significativo do tempo de apoio e, fundamentalmente, com o aumento considerável do tempo de travagem. Desta forma, é possível concluir que a redução do tempo de apoio constitui um factor de rendimento importante (3).

\section{Dinâmica do comportamento angular}

$\mathrm{Na}$ figura 9 estão representadas as curvas médias de posição angular $(\theta)$ para as articulações coxo-femoral, joelho e tíbio-társica. Existe uma diferença clara de comportamento entre a coxo-femoral e as articulações do joelho e tíbio-társica. Estas últimas apresentam um comportamento em ciclo muscular de alongamento encurtamento, enquanto que a articulação coxo-femoral apresenta essencialmente um comportamento de extensão, embora durante a fase inicial do apoio permaneça praticamente inalterada. Este comportamento reforça a ideia de que, a velocidades elevadas, a articulação coxo-femoral do membro inferior em apoio não apresenta acção de flexão $(1,23)$. Contudo, para velocidades de corrida até aproximadamente $8 \mathrm{~ms}^{-1}$, a articulação coxo-femoral, durante um breve período, apresenta um comportamento de flexão, no sentido de amortecer o movimento descendente do corpo (9). 

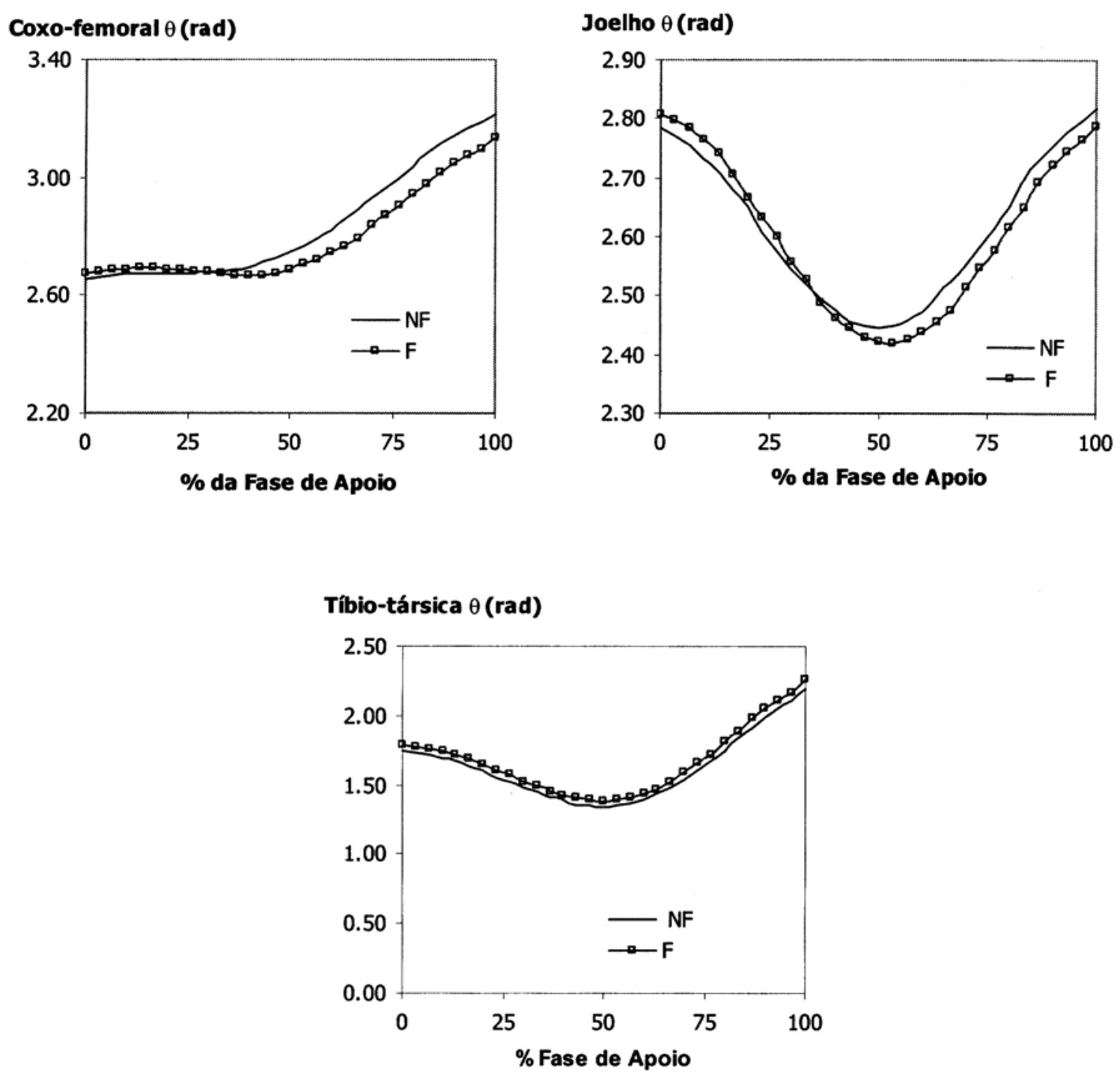

Figura 9 - Curvas médias do deslocamento angular $[\theta$ ] para as articulações coxo-femoral, joelho e tíbio-társica. 0 tempo é expresso em termos de \% da fase de apoio

Esta poderá ser, eventualmente, uma explicação para a ligeira flexão da coxo-femoral verificada em condições de fadiga, já que existe uma redução da velocidade de corrida para valores próximos de $8 \mathrm{~ms}^{-1}$. Uma outra explicação poderá resultar de uma degradação da técnica de transposição, como resultado da instalação da fadiga.
Por sua vez os registos da articulação do joelho e tíbio-társica mostram que os atletas iniciam o apoio com uma flexão destas duas articulações, que mantêm até sensivelmente $48 \%$ do tempo de apoio.

Neste momento alcança-se a maior flexão do joelho, que atinge os 140 graus. Esta flexão segundo Coh e col. (6) é demasiado acentuada, já que os autores 
referem como ângulo mínimo o valor de 147 graus. Contudo, e ainda que não tenham sido encontrados muitos valores de referência para a flexão máxima do joelho num apoio de impulsão para a barreira, existem algumas referências relativamente ao apoio de uma corrida de velocidade. Segundo Tupa e col. (39) a flexão do joelho efectua-se até um ângulo de 140 graus, colidindo com a recomendação de Hoskisson e Korchemny (13) de não baixar mais do que os 145 graus. Por outro lado, Ozolin (34) recomenda para um apoio de corrida uma flexão do joelho de 4 a 10 graus, depois de iniciado o contacto com o solo. No presente estudo, durante a fase de alongamento, o deslocamento angular do joelho variou 19 graus, reflectindo uma superior travagem do movimento, relativamente a um apoio de corrida. Esta variação é ainda mais acentuada quando o apoio é realizado em condições de fadiga. Assim, com a instalação da fadiga a variação angular do joelho na fase de alongamento atinge os 22.6 graus, sugerindo um apoio menos dinâmico e uma menor eficiência na utilização da energia elástica (5).

A articulação tíbio-társica apresenta um padrão de comportamento muito idêntico nas duas situações, embora se verifique, com o aumento da duração da fase de apoio, uma maior extensão desta articulação. A variação angular é, portanto, superior em condições de fadiga, variando entre um ângulo mínimo de 1.38 rad e um ângulo de saída de 2.26 rad.

De referir que as três articulações alcançam as velocidades máximas de extensão antes de atingir a sua extensão máxima, sugerindo um movimento explosivo dos seus músculos extensores. Contudo, em situação de fadiga, e como já foi referido, os picos máximos de velocidade são inferiores e atingidos mais tarde, principalmente ao nível da articulação coxo-femoral, o que denota um movimento menos explosivo e potente.

\section{Dinâmica do comportamento muscular}

A elevada carga a que os músculos são sujeitos durante a fase excêntrica é uma característica importante associada aos movimentos de impulsão máxima. Segundo Komi (17), a capacidade de resistir a essa cargas sugere um alto grau de stiffness muscular nesta fase do ciclo muscular. Ainda segundo o mesmo autor, num apoio de corrida, o tricípite sural apresenta uma actividade eléctrica bastante intensa antes de se iniciar o contacto com o solo, o que permite um nível de tensão durante a fase excêntrica da contracção muscular. Desta forma, o reduzido alongamento verificado no movimento por nós estudado está associado a uma pré-activação muscular, que resulta de um processo de pré-programação do Sistema Nervoso Central (8), preparando o músculo para resistir às cargas de impacto (21), através da ligação de algumas pontes cruzadas entre as proteínas contrácteis.

Por outro lado, segundo Komi e Nicol (19), em condições de fadiga o sistema neuromuscular altera a regulação do stiffness muscular, reflectindo-se numa redução da sensibilidade ao alongamento (2). Desta forma, embora não tenham sido registadas alterações significativas na dimensão do alongamento muscular com a instalação da fadiga, a verdade é que os tempos de acção excêntrica aumentaram, o que sugere uma diminuição da potência mecânica dos músculos extensores do membro inferior, que resulta provavelmente de uma alteração do stiffness muscular. Esta suposição é deduzida a partir da superior variação angular excêntrica do joelho em condições de fadiga e da ligeira diminuição das velocidades angular e de alongamento muscular. Estas condições sugerem uma capacidade diminuída de resistir às cargas de alongamento, uma menor eficiência na utilização da energia elástica e a possibilidade de uma menor contribuição reflexa.

Em síntese, a fadiga associada ao esforço característico dos 400 metros barreiras induz alterações significativas na capacidade de produção mecânica do complexo músculo-tendinoso. Este necessita de mais tempo para atingir idênticos níveis de força, necessidade essa que é traduzida no aumento considerável da duração da fase de apoio, confirmando uma diminuição da capacidade elástica e contráctil muscular. Esta diminuição da capacidade de produção mecânica conduz ao aumento das perdas de velocidade horizontal durante o apoio e, consequentemente, à diminuição da velocidade de deslocamento.

\section{CORRESPONDÊNCIA}

\section{Maria João Valamatos}

Rua D. Mafalda, 19, $7^{\circ}$ Dto, Massamá,

2605-201 Belas, Portugal

mjvalamatos@fmh.utl.pt 


\section{BIBLIOGRAFIA}

1. Atwater, A. E. (1973). Cinematographic analysis of human movement. In J. Wilmore (Ed.) Exercise and sport science reviews, Vol 1. New York: Academic Press, 217-246.

2. Avela, J.; Komi, P. (1998). Reduced stretch reflex sensitivity and muscle stiffness after long-lasting stretch-shortening cycle exercise. Eur J Appl Physiol 78: 403-410.

3. Brejzer V.; Brublevzkij, E. (1984). The 400 Meters Hurdles. Modern Athlete and Coach 22 (3): 32-34

4. Cabri, J. (1989). The influences of different doses alprazolam on muscle activity, in function and cardiovascular responses to concentric and eccentric efforts in isokinetic movement conditions. Doctoral Thesis, Vrije Universiteit Brussel, Brussels.

5. Coh, M.; Dolenec, A. (1996). Three - dimensional Kinematics Analysis of the Hurdles Technique Used By Brigita Bukovec. New Studies in Athletics 11, 1: 63-69.

6. Coh, M.; Kastelic, J.; Pintaric, S. (1998). A Biomechanical Model of the $100 \mathrm{~m}$ Hurdles of Brigita Bukovec. Track Coach 142: 4521-4529.

7. Costa, A. (1996). Caracterização da corrida de 400 metros planos: identificação de algumas variáveis condicionantes do rendimento. Porto: Faculdade de Ciências do Desporto e de Educação Física, Universidade do Porto.

8. Dietz, V.; Noth, J.; Schmidtbleicher, D. (1981). Interaction between pre-activity and stretch reflex in human triceps brachii during landing from forwards falls. J Physiol 311: 113-125.

9. Dillman, C. J. (1975). Kinematic analysis of running. In J. Wilmore \& J. Keogh (Eds.) Exercise and sport sciences reviews (Vol. 3). New York: Academic Press.

10. Gollhofer, A.; Komi, P.V.; Miyashita, M.; Aura, O., (1987). Fatigue during stretch-shortening cycle exercises. I. Changes in mechanical performance of human skeletal muscle. Int J Sports Med 8: 71-78.

11. Grieve, D. W.; Pheasant, S.; Cavagna, G. A. (1978). Prediction of gastrocnemius length from knee and ankle joint posture. In E. Asmussen \& K. Jorgesen (Eds) Biomechanics VI-A. Baltimore: University Park Press, 405412.

12. Horita, T.; Komi, P. V.; Nicol, C.; Kyröläinen, H. (1996). Stretch-shortening cycle fatigue: Interactions among joint stiffness, reflex, and muscle mechanical performance in drop jump. Eur J Appl Physiol 73: 393-403.

13. Hoskisson, J. L.; Korchemny, R. (1991). 1990 TAC junior sprint project stride evaluation. Track Technique 116: 36913699.

14. Jacobs, R.; Bobbert, M. F; van Ingen Schenau, G. (1996). Mechanical output from individual muscles during explosive leg extensions: the role of biarticular muscles.J Biomech 29 (4): 513-523.

15. Jacobs, R.; van Ingen Schenau, G. (1992). Intermuscular coordination in a sprint push-off. J Biomech 25 (9): 953965.

16. Keller, T. S.; Weisberger, A. M.; Ray J. L.; Hasan, S. S.; Shiavi, R.G.; Spengler, D. M. (1996). Relationship between vertical ground reaction force and speed during walking, slow jogging, and running. Clin Biomech (Bristol, Avon) 11(5): 253-259

17. Komi, P. V. (1992). Stretch-Shortening Cycle. In P. V. Komi (Ed.) Strength and Power in Sport. Oxford: Blackwell Scientific Publications, 169-179
18. Komi, P. V.; Bosco, C. (1978). Utilization of stored elastic energy in leg extensor muscles by men and women. Med Sci Sports Exerc 10, 4: 261-267.

19. Komi, P. V.; Nicol, C. (2000). Stretch-Shortening Cycle Fatigue. In B. M. Nigg, B. R. MacIntosh, J. Mester Biomechanics and Biology of Movement. Champaign: Human Kinetics, 385-408.

20. Komi, P. V.; Nicol, C. (2000). Stretch-Shortening Cycle of Muscle Function, in Biomechanics in Sport. Performance enhancement and injury prevention. In V. Zatsiorsky The Encyclopedia of Sport Medicine, Vol IX, 87-102.

21. Kyröläinen, H.; Avela J.; Komi, P. V. (1989). Regulation of muscle force and stiffness during long jump take-off. In $\mathrm{R}$. J. Gregor, R. F. Zernicke \& W. C. Whiting (Ed.) XII International Congress of Biomechanics. Los Angeles: University of California, 219-220.

22. Kyröläinen, H.; Belli, A.; Komi, P. V. (2001). Biomechanical Factors Affecting Running Economy. Med Sci Sports Exerc 33, 8: 1330-1337.

23. Mann, R. (1986). The Biomechanical analysis of sprinting. Track Tecnhique 94: 3000-3003.

24. McDonald, C.; Dapena, J. (1991). Linear Kinematics of the Men's 110-m and Women's 100-m Hurdles Races. Med Sci Sports Exerc 23, 12: 1382-1391.

25. McDonald, C.; Dapena, J. (1991). Angular Momentum in the Men's 110-m and Women's 100-m Hurdles Races. Med Sci Sports Exerc 23, 12: 1392-1402.

26. McLean, B. (1994). The biomechanics of Hurdling: Force Plate Analysis to assess Hurdling Technique. New Studies in Athletics 9, 4: 55-58.

27. Mero, A.; Komi, P. (1986). Force-, EMG-, and elasticityvelocity relationships at submaximal, maximal and supramaximal running speeds in sprinters. Eur J Appl Physiol. Occup. Physiol 55 (5): 553-561.

28. Molina, L. G.; Ozcariz, J. A. (2002). Metodología del Entrenamiento de Velocidad y Vallas en las Categorías de Formación. Apuntes Curso Formación Nivel II - IAAF. "Velocidad y Vallas". Algarve, Nov 2002.

29. Nicol C.; Komi, P. V.; Marconnet, P. (1991). Fatigue effects of marathon running on neuromuscular performance. I. Changes in muscle force and stiffness characteristics. Scand J Med Sci Sports 1: 10-17.

30. Nicol C.; Komi, P. V.; Marconnet, P. (1991). Fatigue effects of marathon running on neuromuscular performance. II. Changes in force, integrated electromyographic activity and endurance capacity. Scand J Med Sci Sports 1: 18-24.

31. Nicol, C.; Komi, P. V.; Marconnet, P. (1991). Effects of marathon fatigue on running kinematics and economy. Scand J Med Sci Sports 1: 195-204.

32. Nilsson J.; Thorstensson A. (1989). Ground reaction forces at different speeds of human walking and running. Acta Physiol Scand 136(2): 217-27

33. Nummela, A.; Rusko, H.; Mero, A. (1994). EMG Activities and Ground Reaction Forces During Fatigued and Nonfatigued Sprinting. Med Sci Sports Exerc 26, 5: 605-609.

34. Ozolin, E. S. (1986). The Sprint. Moscow.

35. Rack, P.M.H.; Westbury, D.R. (1974). The short range elastic stiffness of active mammalian muscle and its effect on mechanical properties. J Physiol 240: 331-350.

36. Santos, P. M-H. (1995). Adaptações Musculares ao Treino da Força. Com especial referência para as adaptações do 
padrão electromiográfico induzidas pelo treino e destreino. Dissertação de doutoramento. Faculdade de Motricidade Humana, Universidade Técnica de Lisboa.

37. Sherman, W. M.; Armstrong, L. E.; Murray, T. M.; Hagerman, F. C.; Costill, D. L.; Staron, R. C.; Ivy, J. L. (1984). Effect of a $42.2 \mathrm{~km}$ footrace and subsequente rest or exercise on muscular strength and work capacity. J Appl Physiol 57 (6): 1668-1673.

38. Sprague, P.; Mann, R. V. (1983). The Effects of Muscular Fatigue on the Kinetics of Sprint Running. Research Quarterly for Exercise and Sport 54, 1: 60-66.

39. Tupa, V.; Gusejnov, F.; Mironenko, I. (1991). Fatigue-induced changes in sprinting technique. Soviet Sport Review, Dec. 185-188.

40. Veloso, A. P., (2000). Biomecânica do comportamento inter-segmentar: modelo do sistema músculo-esquelético e sua aplicação. Dissertação de doutoramento. Faculdade de Motricidade Humana, Universidade Técnica de Lisboa.

41. Visser, J. J.; Hoogkamer, J. E.; Bobbert, M. F.; Huijing, P. A. (1990). Length and moment arm of human leg muscles as a function of knee and hip-joint angles. Eur J Appl Physiol 61: 453-460. 